\title{
The XMM-Newton view of stellar coronae: High-resolution X-ray spectroscopy of Capella ${ }^{\star}$
}

\author{
M. Audard ${ }^{1}$, E. Behar ${ }^{2}$, M. Güdel ${ }^{1}$, A. J. J. Raassen ${ }^{3}$, D. Porquet $^{4}$, R. Mewe ${ }^{3}$, C. R. Foley ${ }^{5}$, \\ and G. E. Bromage ${ }^{6}$ \\ 1 Paul Scherrer Institut, Würenlingen and Villigen, 5232 Villigen PSI, Switzerland \\ 2 Columbia Astrophysics Laboratory, Columbia University, New York, NY 10027, USA \\ 3 SRON Laboratory for Space Research, Sorbonnelaan 2, 3584 CA Utrecht, The Netherlands \\ ${ }^{4}$ CEA/DSM/DAPNIA, Service d'Astrophysique, CEA Saclay, 91191 Gif-sur-Yvette Cedex, France \\ 5 Mullard Space Science Lab., University College London, Surrey RH5 6NT, UK \\ ${ }^{6}$ Centre for Astrophysics, University of Central Lancashire, Preston PR1 2HE, UK
}

Received 2 October 2000 / Accepted 14 November 2000

\begin{abstract}
We present the high-resolution RGS X-ray spectrum of the stellar binary Capella observed by the $X M M$ Newton satellite. A multi-thermal approach has first been applied to fit the data and derive elemental abundances. Using the latter, the emission measure distribution has been reconstructed using a Chebychev polynomial fit. Its shape is found to display a sharp peak around $7 \mathrm{MK}$, consistent with previous $E U V E$ and $A S C A$ results. A smaller but significant amount of emission measure is required around 1.8 MK in order to explain the O viI He-like triplet and the $\mathrm{C}_{\mathrm{Vi}} \mathrm{Ly} \alpha$ line. We have applied the temperature diagnostics of dielectronic recombination satellite lines to the He-like O VII triplet to constrain the cool plasma temperature, and have obtained a lower limit consistent with the global reconstruction of the emission measure distribution. We have used line ratios from the forbidden, intercombination, and resonance lines of the $\mathrm{O}$ VII triplet to derive an average density for the cool coronal plasma $\left(n_{\mathrm{e}}<110^{10} \mathrm{~cm}^{-3}\right)$. Implications for the coronal structure of Capella are discussed.
\end{abstract}

Key words. stars: abundances - stars: activity - stars: coronae - stars: individual: Capella - X-rays: stars

\section{Introduction}

Since the discovery a quarter of a century ago of weak X-ray emission from the first coronal object other than the Sun, Capella (Catura et al. 1975), the solar-stellar connection has been in the focus of the efforts to interpret stellar coronae. Despite the overall similarity of solar and stellar coronae, a number of features set active stellar coronae apart from the solar example. Are stellar coronae scaled-up versions of the solar corona? Is the coronal heating mechanism identical, given the very different average coronal temperatures?

The high X-ray flux of the binary star Capella makes it one of the best candidates to study the X-ray emission from stellar coronae and to identify its role in the larger solar-stellar connection picture. One of the most powerful tools to derive physical properties of stellar coronae is $\mathrm{X}$-ray spectroscopy, providing access to emission measure

Send offprint requests to: M. Audard,

e-mail: audard@astro.phys.ethz.ch

* Based on observations obtained with XMM-Newton, an ESA science mission with instruments and contributions directly funded by ESA Member States and the USA (NASA). distributions in temperature, elemental abundances, densities, and mass motions. Previous X-ray satellites carried low-resolution devices, or if high-resolution was available, then the effective area was small. The advent of a new generation of X-ray satellites, including Chandra and XMMNewton, allows us to obtain high-resolution spectroscopy with moderately high effective areas. We use the Capella spectrum to derive fundamental parameters of its coronae that we compare with previous measurements. The large signal-to-noise ratio of Capella's spectrum allows us to also discuss limitations in the presently available spectral codes.

\section{Previous knowledge on Capella}

Capella ( $\alpha$ Aurigae; 13 Aurigae; HD 34029; HR 1708) is, at a distance of $12.93 \mathrm{pc}$ (Perryman et al. 1997), one of the brightest X-ray objects visible in the sky. It is composed of G1 III + G8 III star (Strassmeier \& Fekel 1990; Hummel et al. 1994) separated by 56.47 mas (Hummel et al. 1994). The orbital period $(P=104 \mathrm{~d})$ is not linked to the rotation period of each component, the G1 giant completing about 12 revolutions in one orbital period 
(Hummel et al. 1994). Catura et al. (1975) first detected weak X-ray emission from Capella, quickly confirmed by Mewe et al. (1975). Subsequent extreme ultraviolet (EUV) and X-ray observations have been prolific with most satellites (e.g., Cash et al. 1978; Holt et al. 1979; Mewe et al. 1982; Vedder \& Canizares 1983; Lemen et al. 1989; Dupree et al. 1993; Schrijver et al. 1995; Favata et al. 1997; Brickhouse et al. 2000; Behar et al. 2000; Brinkman et al. 2000; Canizares et al. 2000), however still leaving unresolved problems in the interpretation of Capella's coronal spectrum.

Linsky et al. (1998) found that the contribution of both stars to the total flux of the coronal Fe Xxi line in the ultraviolet regime was similar. Dupree et al. (1993) showed that iron (FeXV-XXIV) was dominating the EUVE spectrum. Their emission measure $(E M)$ distribution ranged from 0.1 to $63 \mathrm{MK}$, with minimum $E M$ around $1 \mathrm{MK}$ and a sharp peak around 6 MK. Based on lines of highly ionised FexxI, they derived an electron density of $410^{11}-10^{13} \mathrm{~cm}^{-3}$. However, their $E M$ distribution was not in agreement with later BeppoSAX results (Favata et al. 1997). Brickhouse et al. (2000) studied simultaneous EUVE and ASCA observations of Capella. They found that the low first-ionization-potential (FIP) elements Mg, $\mathrm{Si}, \mathrm{S}$, and Fe have coronal abundances consistent with solar photospheric values, while the high-FIP element Ne appears to be underabundant by a factor of 3 to 4 . However, they were not able to constrain the $\mathrm{O}$ abundance, while Brickhouse (1996) derived a subsolar O abundance from the EUVE data. Dupree \& Brickhouse (1996) found a long-term variability in the EUV intensities of Fe XXI to Fe XXIV by up to a factor of 4 .

Recently, first results on Capella were obtained from Chandra HETG/LETG (Behar et al. 2000; Brinkman et al. 2000; Canizares et al. 2000; Mewe et al. 2001b; Ness et al. 2001), confirming the dominance of highly ionised $\mathrm{Fe}$ lines in the X-ray spectrum of Capella. Density diagnostics applied to the $\mathrm{Cv}, \mathrm{N}$ VI and O vII triplets for LETG data implied a low density regime $(2.8 \pm 1.3,6 \pm 3$, and $<5$ times $10^{9} \mathrm{~cm}^{-3}$, respectively). In HETG data, the O vil triplet, formed at low $T$, gave a slightly higher density $\left(0.8-210^{10} \mathrm{~cm}^{-3}\right)$, while the Mg XI and Si XIII triplets, formed at higher $T$, gave upper limits near $710^{11}$ and $110^{12} \mathrm{~cm}^{-3}$. Little (or no) evidence for opacity effects in the $15.014 \AA$ Fe XVII line has been seen. Behar et al. (2000) reproduced fairly well the HETG Fe L-shell spectrum by assuming a single electron temperature of $0.6 \mathrm{keV}$. The present paper presents first results from the observation of Capella with XMM-Newton.

\section{Data reduction and analysis}

Capella was observed several times by XMM-Newton (Jansen et al. 2001) for calibration purposes. In this Letter, we present the Reflection Grating Spectrometer (RGS; den Herder et al. 2001) data of the on-axis observation (2000-03-25, 11:36:59 UT until 2000-03-26, 02:53:49 UT) which had a low instrumental background. The effective
Table 1. Line fluxes in $10^{-4} \mathrm{~s}^{-1} \mathrm{~cm}^{-2}$. RGS2 fluxes (except for O VII lines only available in the RGS1 spectrum) are compared to Chandra HETG and LETG measurements

\begin{tabular}{lllll}
\hline Ion & $\lambda(\AA)$ & RGS & HETG $^{\mathrm{a}}$ & LETG $^{\mathrm{b}}$ \\
\hline Mg XII & 8.421 & $2.2 \pm 0.4$ & 1.5 & $2.0 \pm 0.2$ \\
Mg XI & 9.169 & $3.9 \pm 0.6$ & 3.5 & $3.2 \pm 0.3$ \\
Mg XI & 9.231 & $0.9 \pm 0.3$ & 0.6 & $0.7 \pm 0.2$ \\
Mg XI & 9.314 & $1.5 \pm 0.3$ & 1.9 & $1.6 \pm 0.2$ \\
Fe XVII & 15.014 & $37.9 \pm 1.7$ & 30.4 & $34.1 \pm 1.1$ \\
Fe XVII & 16.775 & $23.1 \pm 1.3$ & 20.0 & $20.4 \pm 0.8$ \\
Fe XVII & 17.051 & $26.4 \pm 1.5$ & 26.4 & $30.9 \pm 1.9$ \\
Fe XVII & 17.100 & $31.1 \pm 1.6$ & 24.4 & $24.4 \pm 3.0$ \\
O VIII & 18.969 & $26.8 \pm 1.5$ & 26.3 & $26.4 \pm 1.0$ \\
O VII & 21.602 & $7.6 \pm 0.8$ & 9.7 & $9.9 \pm 0.7$ \\
O VII & 21.804 & $1.1 \pm 0.3$ & 2.6 & $1.9 \pm 0.3$ \\
O VII & 22.101 & $4.8 \pm 0.6$ & 7.4 & $6.9 \pm 0.6$ \\
N VII & 24.781 & $6.3 \pm 0.7$ & 5.5 & $6.9 \pm 0.8$ \\
C VI & 33.734 & $7.9 \pm 0.8$ & & $8.7 \pm 0.9$ \\
\hline
\end{tabular}

${ }^{\mathrm{a}}$ Canizares et al. (2000), b Mewe et al. (2001b).

exposure times were 52.3 and $52.4 \mathrm{ksec}$ for RGS1 and RGS2, respectively. The data from the other X-ray instruments onboard XMM-Newton could not be used, because they were severely piled-up and optically contaminated by the brightness of Capella.

The data were analysed with the official ESA XMM Science Analysis System (SAS) software, version 4.1, and an update of several RGS tasks together with the latest calibration files available at the time of the analysis. The metatask RGSPROC 0.77 was used to process the RGS data. Spectra were extracted along the dispersion direction using a spatial mask together with a cut in the plane of dispersion angle vs. CCD energy. The satellite pointing was stable, except for a short (600 s) deviation of the attitude pointing that had no significant influence on the RGS spectra of Capella. The RGS response matrices were generated by RGSRMFGEN 0.29. Although the detailed description of the response of both spectrometers is expected to evolve over time, we should note that the description of the RGS2 response is more advanced than for RGS1. Some systematic errors may be introduced for RGS1 results. However, any future RGS analysis with a more advanced response is not expected to give results that deviate more than $\approx 10-25 \%$ from the current results.

\subsection{Line fluxes}

A number of individual line fluxes have been measured in the RGS spectra (Table 1).

First, a constant "background" level was adjusted in order to account for the real continuum or for the pseudo-continuum created by the overlap of several weak or neglected lines. Especially in the 9-18 $\AA$ region, the overlap of the line wings (originating from the instrumental line spread function, LSF) created an important 

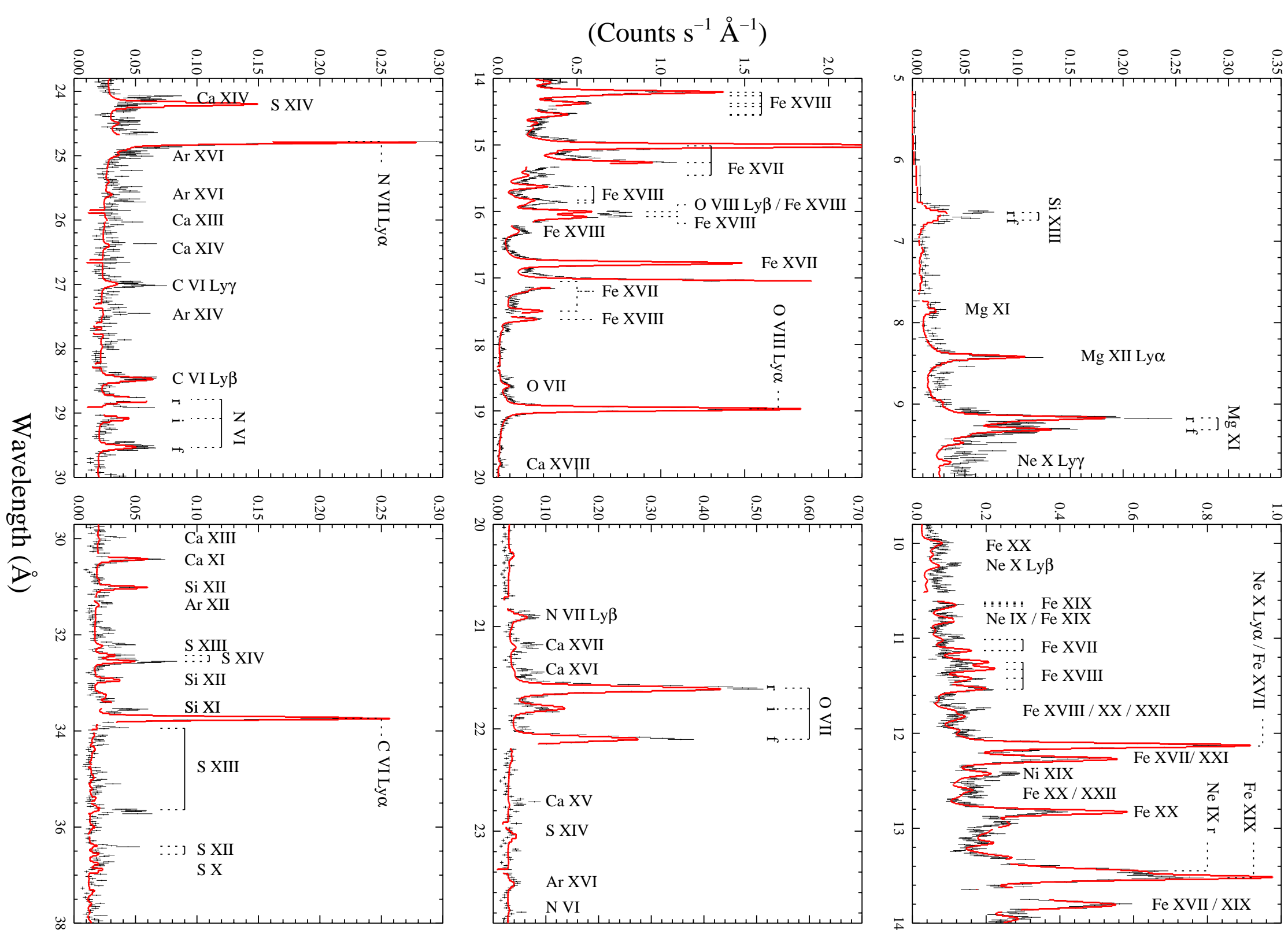

\section{Letter to the Editor}


pseudo-continuum. We used the instrumental LSF in order to derive the integrated line fluxes. For comparison, we also give line fluxes as measured by Chandra HETG (Canizares et al. 2000) and LETG (Mewe et al. 2001b). Within the instrumental and calibration uncertainties, the RGS fluxes are similar to the non-simultaneously measured Chandra fluxes. There may be some indications for higher flux values in Chandra for lower stages of ionization. This could also suggest that the low temperature plasma has lower EM during the XMM-Newton observation. This is, however, opposite to the suggestion of Dupree \& Brickhouse (1996) who found flux variations in the hot plasma component.

\subsection{Working procedure}

In this Letter, we have used a "global fitting" approach. This approach allows us to obtain a self-consistent solution that best fits the data and that takes into account simultaneously the contributions of the continuum components and of all emission lines. Line blends and the overlap of line wings are therefore fully accounted for, and the line fluxes are correctly reproduced within the accuracy of the code. However, as will be discussed in the following sections, the fit depends on the completeness of the atomic database used by the spectral fitting code. We have used the publicly available Utrecht software SPEX 2.0 (Kaastra et al. 1996). It contains a collisional ionization equilibrium (CIE) model that is equivalent to the MEKAL (Mewe et al. 1995) code available in the XSPEC software (Arnaud 1996), except that the former includes a significant update of the wavelengths of Fe L-shell lines between 10-18 $\AA$, based on the solar data by Phillips et al. (1999).

A spectral model using three CIE components with a column density $N_{\mathrm{H}}=1.810^{18} \mathrm{~cm}^{-2}$ (Linsky et al. 1993) allowed us to obtain an initial representation of the temperature structure in Capella. This model was used to derive coronal elemental abundances from a variety of bright and weak lines. We added further components (up to ten) in the multi- $T$ approach to investigate whether they are required for a better description of the fit parameters. This was not the case. We therefore interpret our abundances as sufficiently accurate within the limitations of the spectral code. The best-fit results are given in Table 2 for RGS2 data, and for completeness also for RGS1. The uncertainties do not include systematic uncertainties that may be introduced by calibration uncertainties (especially for RGS1, see Sect. 3). A continuous emission measure distribution has then been reconstructed using the Chebychev polynomial fit approach, using the best-fit coronal elemental abundances obtained from the 3-T CIE model.

The very high signal-to-noise ratio of the RGS spectra of Capella makes visible several places where the spectral code systematically over- or underestimates the data. Since these deviations are systematic and localized, we do not give $\chi^{2}$ values. A formally (based on $\chi^{2}$ ) inacceptable fit does not imply, in this case, that the global fit is
Table 2. Best fits for a $3-T$ CIE model with $90 \%$ confidence ranges for a single parameter. The elemental abundances are given relative to the solar photospheric value (Anders \& Grevesse 1989). See text for a discussion on the significance of the elemental abundances

\begin{tabular}{lll}
\hline Parameter & RGS1 & RGS2 \\
\hline $\log N_{\mathrm{H}}\left[\mathrm{cm}^{-2}\right]$ & $=18.255$ & $=18.255$ \\
$k T_{1}[\mathrm{keV}]$ & $0.159_{-0.006}^{+0.006}$ & $0.13_{-0.01}^{+0.01}$ \\
$k T_{2}[\mathrm{keV}]$ & $0.593_{-0.002}^{+0.002}$ & $0.58_{-0.004}^{+0.003}$ \\
$k T_{3}[\mathrm{keV}]$ & $1.14_{-0.07}^{+0.07}$ & $0.93_{-0.07}^{+0.07}$ \\
$\log E M_{1}\left[\mathrm{~cm}^{-3}\right]$ & $51.90_{-0.02}^{+0.03}$ & $51.65_{-0.09}^{+0.08}$ \\
$\log E M_{2}\left[\mathrm{~cm}^{-3}\right]$ & $52.86_{-0.01}^{+0.009}$ & $52.98_{-0.01}^{+0.01}$ \\
$\log E M_{3}\left[\mathrm{~cm}^{-3}\right]$ & $51.81_{-0.05}^{+0.05}$ & $51.85_{-0.09}^{+0.08}$ \\
$\mathrm{C}$ & $0.50_{-0.04}^{+0.04}$ & $0.52_{-0.04}^{+0.04}$ \\
$\mathrm{~N}$ & $0.82_{-0.07}^{+0.07}$ & $1.01_{-0.08}^{+0.08}$ \\
$\mathrm{O}$ & $0.44_{-0.02}^{+0.02}$ & $0.39_{-0.01}^{+0.01}$ \\
$\mathrm{Ne}$ & $0.51_{-0.03}^{+0.03}$ & $0.34_{-0.02}^{+0.02}$ \\
$\mathrm{Mg}$ & $1.13_{-0.05}^{+0.06}$ & $0.74_{-0.04}^{+0.04}$ \\
$\mathrm{Si}$ & $0.41_{-0.06}^{+0.06}$ & $0.48_{-0.06}^{+0.06}$ \\
$\mathrm{~S}$ & $0.14_{-0.02}^{+0.02}$ & $0.09_{-0.02}^{+0.02}$ \\
$\mathrm{Ar}$ & $0.23_{-0.1}^{+0.1}$ & $0.17_{-0.14}^{+0.14}$ \\
$\mathrm{Ca}$ & $0.27_{-0.08}^{+0.08}$ & $0.27_{-0.11}^{+0.11}$ \\
$\mathrm{Fe}$ & $0.62_{-0.01}^{+0.02}$ & $0.51_{-0.01}^{+0.01}$ \\
$\mathrm{Ni}$ & $0.88_{-0.07}^{+0.07}$ & $0.54_{-0.05}^{+0.05}$ \\
\hline
\end{tabular}

overall incorrect, but rather that there are well-identified local problems with separate lines. Figure 1 shows a model fit to the Capella spectrum.

\subsection{Elemental abundances}

We estimate, in the following, the influence of the fitting discrepancies on our results. The most important discrepancies occur for the Si XIII lines at $6.6 \AA$, for the Fe XVII-XX lines at $11.0-11.4 \AA$, at $12.8 \AA$, at $13.5 \AA$, and for the FexVIII lines around $16 \AA$. The spectral code underestimates the flux around 9.6-10.6 $\AA$, which has been interpreted by Brickhouse et al. (2000) as being due to missing high excitation $(n>5)$ lines of Fe XVII-XIX. Furthermore, the energy dependency of the collision strengths of Fe L-shell lines needs to be updated in SPEX/MEKAL. Additionally, we note that the spectral code fails to pick up some of the weak lines observed in the long-wavelength part of the spectrum. HULLAC (Bar-Shalom et al. 1998) calculations indicate that these should mostly be attributed to L-shell emission from Si, $\mathrm{S}$, Ar, and Ca. The mentioned discrepancies are related to the incompleteness of the atomic database of the CIE model in SPEX, and similarly of the MEKAL model in XSPEC. We conclude that L-shell lines from several elements (e.g., S, Si, Ca, Ar, Ni) and high excitation Fe L-shell lines are insufficiently described in these codes.

To test the robustness of the derived elemental abundances, we have iteratively eliminated emission lines and parts of the continuum that showed poor fit results, thus moving from global fitting towards a "single line analysis" approach. As an aside, we note here that the overlapping 


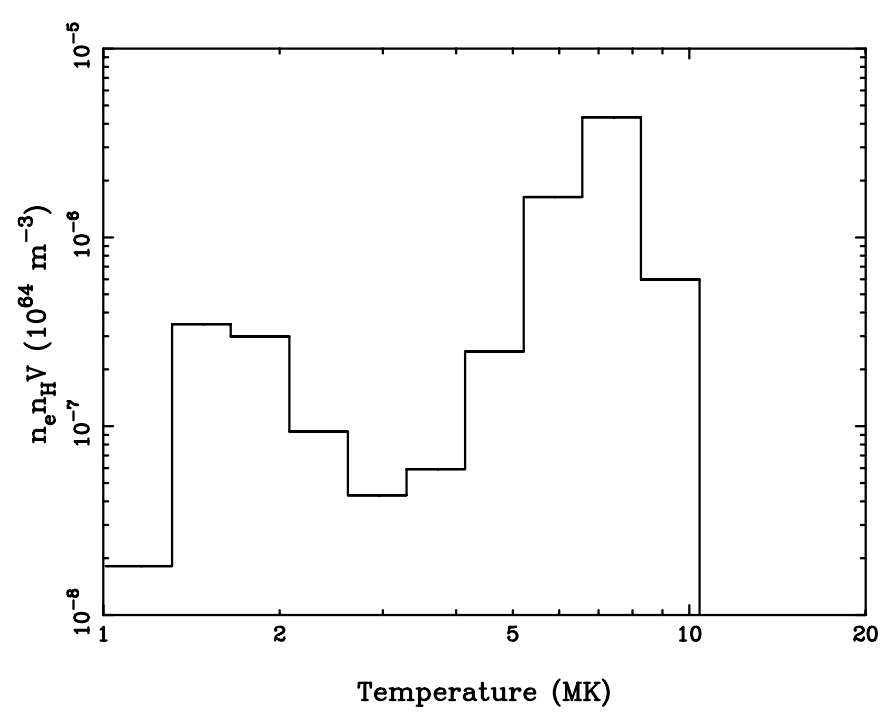

Fig. 2. Realization of the $E M$ distribution using Chebychev polynomials of order 5 . The distribution does not show any significant $E M$ above $10 \mathrm{MK}$

line wings as well as several line blends in the present RGS spectrum introduce considerable uncertainty if single line fluxes in the Fe L-shell region are measured without modeling; we therefore kept the "global" approach for this test, even when eventually only a few bright line lines contributed to the results. Despite the large reduction of the spectral information, the abundances turned out to be quite robust. In all test runs, the $\mathrm{Fe}$ abundance was confined to within $0.50-0.68$ (times the solar photospheric value), $\mathrm{O}$ within $0.24-0.39$, and $\mathrm{Ne}$ within $0.75-1.05$. The $\mathrm{S}$ abundance is basically derived from weak L-shell lines in the RGS band. The determination of its abundance is mostly influenced by the SXIV line at $24.2 \AA$ in the model that is not as strong in the data. When the data around this line are removed, the abundance increases to $0.35 \pm 0.04$, and the model better fits the remaining weak $\mathrm{S}$ lines. Similarly, by removing L-shell Si ions (in the long wavelength band), the bright Si He-like triplet is correctly fitted, with an abundance of 0.7-1.0 times the solar photospheric value.

\subsection{Emission measure distribution}

Using coronal elemental abundances derived from the 3$T$ model, the $E M$ distribution has been self-consistently reconstructed. Figure 2 shows one realisation of the $E M$ distribution using a Chebychev polynomial of order 5 . No $E M$ above $10 \mathrm{MK}$ is needed for this realisation. A sharp peak around 7 MK dominates the spectral emission lines, similar to previous results (Dupree et al. 1993; Schrijver et al. 1995; Brickhouse et al. 2000) and recent results from Chandra (Behar et al. 2000). However, the approximate upper envelope to the true $E M$ distribution found by Canizares et al. (2000) does not reproduce the sharp peak, but indicates a broad distribution of temperature.
It is possible that their assumption of solar abundances explains the discrepancy. However, we note that our EM distributions bears similarity to the distribution derived by Mewe et al. (2001b) from Chandra LETG data.

\subsection{He-like ions}

Using the line ratios $R=f / i$ and $G=(i+f) / r$ (see, e.g., Gabriel \& Jordan 1969; Pradhan 1982) from the fluxes of the resonance line $(r)$, the intercombination line $(i)$ and the forbidden line $(f)$ of the O VII and, tentatively, $\mathrm{Mg}$ XI triplets, we have derived average coronal densities. The $\mathrm{Si}$ triplet is strongly blended because of the decreasing spectral resolution of the RGS at short wavelengths, while the Ne triplet is heavily blended by Fe and Ni lines. For the weak N vi triplet, the line intensities were not unambiguously determined. Note that due to the spatially unresolved nature of stellar coronal X-ray emission, any derived value of a density should be considered as a weighted average of the densities in the various regions of both coronae of Capella.

We used theoretical calculations (Mewe et al. 2001a) that take into account the radiative and dielectronic recombinations, and the electronic collisional excitations (see Porquet \& Dubau 2000 for the atomic data); they also take into account the influence of the radiation field (photo-excitation) which is important for low- $Z$ ions. We refer to Ness et al. (2001) for additional details. From the measured RGS line fluxes (Table 1), the ratios for O VII are $R_{\text {obs }}=4.36 \pm 1.3$ and $G_{\text {obs }}=0.78 \pm 0.12$, implying an average electron temperature of $T \approx 2.7 \mathrm{MK}$ and an upper limit for the average density of $<110^{10} \mathrm{~cm}^{-3}$. For the $\mathrm{Mg}$ triplet, we tentatively get $R_{\mathrm{obs}}=1.67 \pm 0.65$ and $G_{\text {obs }}=0.62 \pm 0.14$, leading to $T \approx 7 \mathrm{MK}$ and $n_{\mathrm{e}}=7_{-3}^{+18} 10^{12} \mathrm{~cm}^{-3}$. However, due to the low spectral resolution at short wavelengths in the RGS (e.g., compared to HETG), the Mg triplet line fluxes are difficult to measure, therefore the derived density should be taken with caution.

\subsection{Dielectronic recombination satellite lines}

The O VII spectral region has been separately investigated for the presence or absence of dielectronic recombination (DR) satellite lines. DR satellite lines of He-like spectral lines in hot collisional plasmas are excellent additional tracers of the cool $(<1 \mathrm{MK})$ plasma otherwise not sufficiently constrained by the available lines. It is, to our knowledge, the first time that such diagnostics is applied to the X-ray spectrum of a stellar corona. The SPEX (and MEKAL) spectral code does presently not include a sufficient description of the DR satellite lines for low- $Z$ ions such $\mathrm{O}$. Therefore, the lower temperature component of the $E M$ distribution is not well constrained below $1 \mathrm{MK}$. Using the HULLAC code, we have calculated the emitted spectrum of the He-like lines of $\mathrm{O}$ VII including the O VI DR satellite lines in the low-density limit. All of 


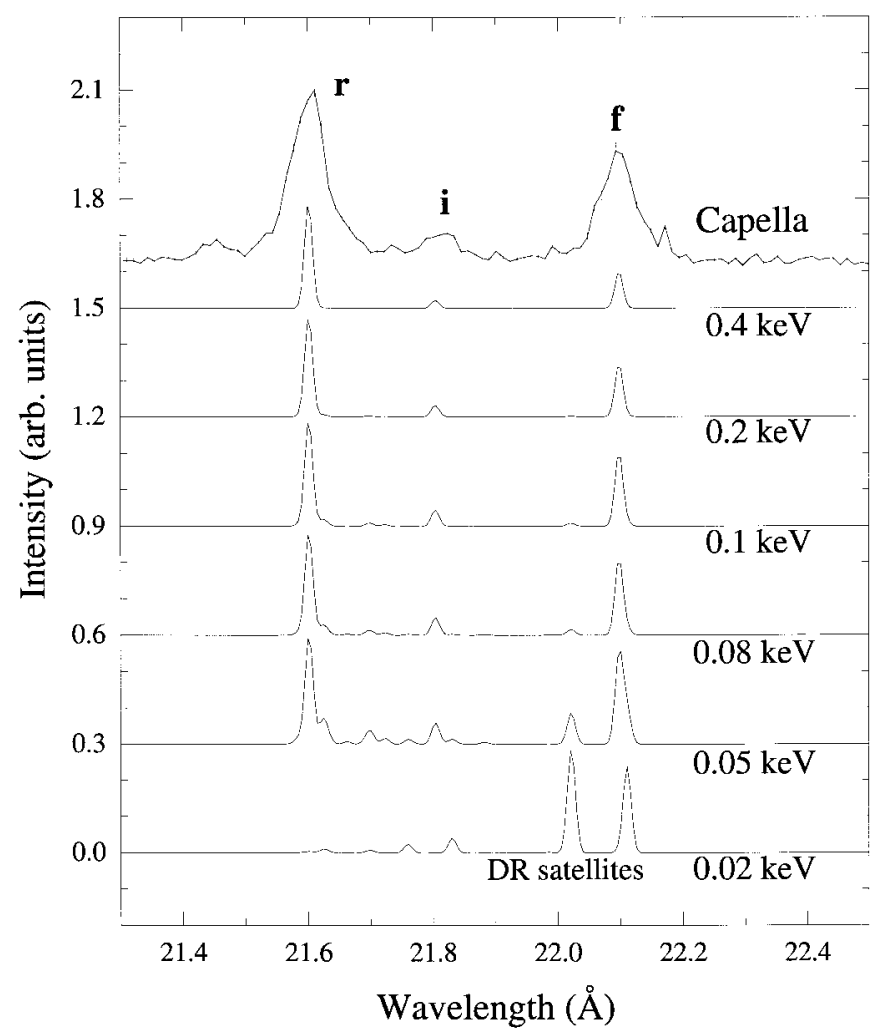

Fig. 3. Observed O VII triplet with calculated spectra of the $\mathrm{O}$ VII and O VI DR satellite lines for six different electron temperatures. The plots are normalized to the strongest line in each spectrum. Note the steep temperature sensitivity of the DR satellites (see text)

the 1 snl and $1 \mathrm{~s} 2 \ln ^{\prime} l^{\prime}\left(n^{\prime}=2\right.$ to 4$)$ levels are included in the computations. The resulting theoretical $\mathrm{O}$ spectra, as a function of electron temperature, are depicted in Fig. 3 together with the data. The strongest DR satellites are at $22.02 \AA$ and at $22.11 \AA$ (Gabriel 1972). The bottom plot $(0.02 \mathrm{keV})$ in the figure clearly demonstrates how the DR satellites dominate the spectrum at low temperature. At slightly higher temperature $(0.05 \mathrm{keV})$, the higher lying satellites, in particular $1 \mathrm{~s} 2 l 3 l^{\prime}$, produce relatively strong lines at $21.70 \AA$ and at $21.63 \AA$, but eventually, as the temperature increases, the DR lines become weak and the He-like triplet lines prevail. The RGS data do not allow the identification of DR satellite lines, setting a lower limit of $\approx 1.2 \mathrm{MK}$ to the temperature of the cooler plasma component that forms the $\mathrm{O}$ VI-VII lines.

\section{Discussion and conclusions}

The high-resolution, high signal-to-noise X-ray spectrum of Capella measured by the RGS instruments on board the $X M M$-Newton satellite offers unprecedented spectroscopic power for the study of stellar coronal plasma. It also gives insight into some deficiencies in the present spectral codes. The X-ray spectra collected from the new generation of X-ray satellites, such as XMM-Newton and Chandra, will put further strong constraints on these codes.
The derived elemental abundances from the Capella RGS spectrum are found to be generally below their solar photospheric values, or close to them. The abundances are generally similar to the abundances derived from a continuous-temperature model applied simultaneously on ASCA and EUVE data (Brickhouse et al. 2000, their Table 4). For the $\mathrm{O}$ and $\mathrm{Ar}$ abundances, we refer to Brickhouse (1996) also reported in the 2nd column of Table 4 in Brickhouse et al. 2000).

Recent analysis of the RGS spectrum of the active RS CVn binary star HR 1099 (Brinkman et al. 2001) suggested the presence of an inverse FIP effect, i.e. elements with a high FIP are overabundant with respect to their photospheric abundances. In Capella, we do not find a clear distinction between the coronal abundances of lowFIP and high-FIP elements. The high-FIP elements Ne, $\mathrm{O}$, and $\mathrm{C}$ have abundances below the solar photospheric values; N, another high-FIP element, is consistent with its solar abundance. Low-FIP elements such as Fe, Si, and $\mathrm{Mg}$ have coronal abundances also slightly below solar, or consistent with solar. The high Fe abundance $(\approx 0.5)$ sets Capella apart from other active stars that typically have Fe abundances well below (0.1-0.3) the solar photospheric value (e.g., Audard et al. 2001; Güdel et al. 2001a, 2001b). It is important to note that McWilliam (1990) derived a photospheric Fe abundance for Capella of 0.43 times solar photospheric, with large errors, however. This may indicate that the coronal Fe abundance is indeed comparable with Capella's photospheric abundance.

The $\mathrm{S}$ abundance $(\approx 0.1$, but $\approx 0.35$ when the data around the S XIV line at $24.2 \AA$ is removed) is much lower than the abundance derived by Brickhouse (1996) and Brickhouse et al. (2000) who found consistency with the solar photospheric abundance. Note that the RGS spectrum only contains weak L-shell lines of S. We believe that the incompleteness of the atomic database for these lines largely influences the determination of the coronal abundance of S. Similarly, the Ar, Ca and Ni abundances should be taken with caution.

A realisation of the $E M$ distribution (Fig. 2) shows that it is continuous but a sharp peak is found between 5-8 MK. Such a peak was suggested from previous EUVE data (Dupree et al. 1993; Schrijver et al. 1995; Brickhouse et al. 2000) and recent Chandra LETG (Mewe et al. 2001b), and HETG data (Behar et al. 2000). Another peak is found at lower temperatures $(1.2-2.5 \mathrm{MK})$, albeit with a lower EM. This bump is mainly required in order to account for the bright emission lines from the relatively cool O vir triplet and C vi Ly $\alpha$.

For the first time, to our knowledge, in a stellar X-ray spectrum, we have used the information from the dielectronic satellite lines of the He-like O viI triplet to constrain the plasma $E M$. Such DR lines are very sensitive to the lowest temperature $(<1 \mathrm{MK})$ coronal components of the plasma. We did not detect these lines, setting a lower limit of $\approx 1.2 \mathrm{MK}$ for the plasma forming $\mathrm{O}$ VI-VII lines, consistent with the lowest temperature with "significant" $E M$ in the globally derived EM distribution. 
Best-fit densities from the "cool" O vil and "hot" Mg XI triplets appear to differ considerably, the former indicating an upper limit of $10^{10} \mathrm{~cm}^{-3}$, consistent with both LETG (Brinkman et al. 2000; Ness et al. 2001) and HETG results (Canizares et al. 2000), and the latter tentatively indicating $10^{12}-10^{13} \mathrm{~cm}^{-3}$. This is higher than a recent estimate of the density derived for $\mathrm{Mg}$ by Canizares et al. (2000). However, Dupree et al. (1993) derived a compatible electron density from the highly ionised Fe XXI.

If the above density values are confirmed, it then appears that the coronae of Capella may be bi-modal: on the one hand, we find a low-density cool plasma, and on the other hand, we measure high densities for the hot plasma. Because it is likely that there is a broad distribution of electron densities in the coronae of Capella, we cannot exclude the presence of cool high-density and hot low-density material. However, it is probable that the two detected plasma portions belong to distinct and different features in the stellar coronae, given the magnitude of their density and temperature differences. This would suggest the simultaneous presence of low-lying, compact hot loops and of larger, cooler loops in the stellar coronal atmospheres of Capella (Mewe et al. 2001b).

Our observations suggest clear limitations for the applicability of solar coronal concepts to more active stars. Although Capella is intermediate in its activity between the Sun and extremely active stars such as HR 1099 or dMe stars, its abundance pattern is neither reminiscent of the latter nor in any way similar to the average solar corona (FIP effect). To uncover systematic trends, it will be important to survey the complete range of activity between inactive, solar-like stars and stars like Capella with further high-resolution spectroscopic observations.

Acknowledgements. MA acknowledges support from the Swiss National Science Foundation (grants 2100-049343 and 2000058827), from the Swiss Academy of Sciences and from the Swiss Commission for Space Research. The Space Research Organization Netherlands (SRON) is supported financially by NWO. CRF acknowledges financial support from the UK Particle Physics and Astronomy Research Council.

\section{References}

Anders, E., \& Grevesse, N. 1989, Geochim. Cosmochim. Acta, 53, 197

Arnaud, K. A. 1996, in ASP Conf. Ser. 101, Astronomical Data Analysis Software and Systems V, ed. G. Jacoby \& J. Barnes (San Francisco: ASP), 17

Audard, M., Güdel, M., \& Mewe, R. 2001, A\&A, 365, L318

Bar-Shalom, A., Klapisch, M., Goldstein, W. H., \& Oreg, J. 1998, The HULLAC code for atomic physics, unpublished

Behar, E., Cottam, J., \& Kahn, S. M. 2000, ApJ, in press [astro-ph/0003099]

Brickhouse, N. S. 1996, in IAU Colloq. 152, Astrophysics in the Extreme Ultraviolet, ed. S. Bowyer \& R. F. Malina (Dordrecht: Kluwer), 105

Brickhouse, N. S., Dupree, A. K., Edgar, R. J., et al. 2000, ApJ, 530, 387
Brinkman, A. C., Behar, E., Güdel, M., et al. 2001, A\&A, 365, L324

Brinkman, A. C., Gunsing, C. J. T., Kaastra, J. S., et al. 2000, ApJ, 530, L111

Canizares, C. R., Huenemoerder, D. P., Davis, D. S., et al. 2000, ApJ, 539, L41

Cash, W., Bowyer, S., Charles, P. A., et al. 1978, ApJ, 223, L21

Catura, R. C., Acton, L. W., \& Johnson, H. M. 1975, ApJ, 196, L47

Dupree, A. K., \& Brickhouse, N. S. 1996, in Poster Proc., IAU Symp. 176: Stellar Surface Structure (Wien: Institut für Astronomie), 184

Dupree, A. K., Brickhouse, N. S., Doschek, G. A., Green, J. C., \& Raymond, J. C. 1993, ApJ, 418, L41

Favata, F., Mewe, R., Brickhouse, N. S., et al. 1997, A\&A, 324, L37

Gabriel, A. H. 1972, MNRAS, 160, 99

Gabriel, A. H, \& Jordan, C. 1969, MNRAS, 145, 241

Güdel, M., Audard, M., Briggs, K., et al. 2001a, A\&A, 365, L336

Güdel, M., Audard, M., Magee, H., et al. 2001b, A\&A, 365, L344

den Herder, J. W., Brinkman, A. C., Kahn, S. M., et al. 2001, A\&A, 365, L7

Holt, S. S., White, N. E., Becker, R. H., et al. 1979, ApJ, 234, L65

Hummel, C. A., Armstrong, J. T., Quirrenbach, A., et al. 1994, AJ, 107, 1859

Jansen, F., Lumb, D. H., Altieri, B., et al. 2001, A\&A, 365, L1

Kaastra, J. S., Mewe, R., \& Nieuwenhuijzen, H. 1996, in UV and X-ray Spectroscopy of Astrophysical and Laboratory, ed. K. Yamashita \& T. Watanabe (Tokyo: Univ. Acad. Press), 411

Lemen, J. R., Mewe, R., Schrijver, C. J., \& Fludra, A. 1989, ApJ, 341, 474

Linsky, J. L, Brown, A., Gayley, K., et al. 1993, ApJ, 402, 694

Linsky, J. L., Wood, B. E., Brown, A., Osten, R. A. 1998, ApJ, 492,767

McWilliam, A. 1990, ApJS, 74, 1075

Mewe, R., Gronenschild, E. H. B. M., Westergaard, N. J., et al. 1982, ApJ, 260, 233

Mewe, R., Heise, J., Gronenschild, E. H. B. M., et al. 1975, ApJ, 202, L67

Mewe, R., Kaastra, J. S., \& Liedahl, D. A. 1995, Legacy, 6, 16

Mewe, R., Porquet, D., Raassen, A. J. J., Kaastra, J. S., \& Dubau, J. 2001a, in preparation

Mewe, R., Raassen, A. J. J., Drake, J. J., Kaastra, J. S., van der Meer, R. L. J., \& Porquet, D. 2001b, A\&A, submitted

Ness, J. U., Mewe, R., Schmitt, J. H. M. M., et al. 2001, A\&A, submitted

Perryman, M. A. C., Lindegren, L., Kovalevsky, J., et al. 1997, A\&A, 323, L49

Phillips, K. J. H., Mewe, R., Harra-Murnion, L. K., et al. 1999, A\&AS, 138, 381

Porquet, D., \& Dubau, J. 2000, A\&AS, 143, 495

Pradhan, A. K. 1982, ApJ, 263, 477

Schrijver, C. J., Mewe, R., van den Oord, G. H. J., \& Kaastra, J. S. 1995, A\&A, 302, 438

Strassmeier, K. G., \& Fekel F. C. 1990, A\&A, 230, 389

Vedder, P. W., \& Canizares, C. R. 1983, ApJ, 270, 666 